\title{
Epilogue: How Should Clinicians Implement the Complex Innate Immune Effector Responses at the Patient's Bedside?
}

The various facets of innate immune effector responses described in this part, when getting uncontrolled and dysregulated, let intuit their great impact on future modern medicine. Nevertheless, most of these responses are complex and complicated, and, thus, their proper implementation by clinicians into innovative diagnostic and therapeutic procedures will be a challenge. For example, how to translate the fundamental knowledge about cell stress/tissue injury-induced activation and function of the innate immune system into the development of new diagnostic and therapeutic approaches for diseases associated with inflammatory features? Indeed, what appears to be already clear is the distinction between suppression and promotion of DAMP-induced innate immune processes: therapeutic downregulation of DAMPs in case of hyperinflammatory responses such as ARDS, SIRS, and sepsis and therapeutic upregulation in case of inferior inflammatory responses, for example, as observed in delayed wound healing. What also seems to be plausible is to use DAMPs as biomarkers for diagnostic and prognostic assessments. Other issues are far less clear, for example, when and where within the sequelae of specific DAMPinduced PRM-triggered inflammatory pathways to interfere? What exact molecules, that is, receptors, transcription factors, and other molecules of a distinct pathway, should be targeted? How to differentiate exactly between an ongoing innate immune inflammatory response still serving the restoration of homeostasis and a response that has already changed to an uncontrolled dangerous pathological reaction? As always in the long history of medicine, the proper answer to all these questions will come from clinical trials which are expected to be designed, conducted, and reported to the medical community in the near future. Volume 2 of the book will deal with these and many more other issues. 